\title{
Editorial
}

Greetings to all our Readers and Authors! The Bangladesh Journal of Bioethics is gaining more popularity among all of us who are concerned about standards, norms, practices and ethics. This journal is our voice to share what is being practiced and what should be done. It will help to bring about equality and equity through an ethical rights based approach in all sectors of development touching our lives.

This issue contains articles on diverse aspects of medical and social practices and will be very useful in ensuring that Bioethical standards are complied with. Some of the papers are discussed below:

Albert M. E. Coleman in his paper discusses the challenges and implications of medical ethics and medical professionalism in low and middle income (LAMIC) countries. The article examines the ethical issues that may arise with regard to medical practice in low and middle income countries (LAMIC), and the challenges for medical regulatory bodies in upholding ethics in professional practice. The Author recommends that LAMIC progress to improve the health of its population should synchronize with medical ethics theories.

Tahera Ahmed in her paper on Child Marriage points out that it is still a massive problem in many developing countries. The paper reviews the current situation and existing programmes on reduction of child marriage. The paper discusses the consequences of child marriage such as risk of early and unwanted pregnancies, maternal morbidities and mortalities. The Author recommends that each country set up its own mid-term and long-term goals to bring about significant reduction in child marriages.

Dr. Mohammad Arifur Rahman et al looks into the influence of drugs and device industries on healthcare research, and practice. The authors through literature review and analysis have presented their findings at the following levels: (i) evidence base production, (ii) evidence synthesis, (iii) understanding of safety and harms issues, (iv) costeffectiveness evaluation, (v) clinical practice guidelines formation, (vi) healthcare professional education, (vii) healthcare practice, (viii) healthcare consumer's decisions. They conclude that the industry influences evidence base production, evidence synthesis, understanding of harms issues, cost-effectiveness evaluations, clinical practice guidelines and Healthcare professional education and exerts direct influence on professional decisions and health consumers. The authors recommend the need for regulation of medicine towards a more beneficial direction free from conflict of interests.

Zoheb Rafique presents a case study relating to Accountability for Reasonableness, for Priority Setting and Resource Allocation. Through a case study he examines the priority setting and resource allocation for scarce resources in a developing country like Pakistan. He applies the four conditions of accountability for reasonableness for priority setting and resource allocation. These four conditions are publicity condition, relevance condition, revisions and appeals condition and regulative condition. In resource poor settings the four conditions of 'Accountability for reasonableness' is a framework that can be used to guide priority setting in health care organizations. The author also recommends that more hospitals be set up, doctors trained, equipment and supplies provided. He concludes that even with scarce resources, the government hospitals in Pakistan are providing excellent services.

Dear Readers, your articles, reports or commentaries are welcome! In whatever field you are working in, Bioethics play an important role, and we hope that through this journal you will share your thoughts and experiences for us to build a better and improved quality of life.

\section{Best Regards.}

\section{Tahera Ahmed}

Former Chief, Sexual and Reproductive Health, UNFPA, Faculty, North South University,

And

Editor, Bangladesh Journal of Bioethics 